\title{
Research on hull segment workshop scheduling based on simulated annealing genetic algorithm
}

\author{
Fei LI, Xiaoping MA
}

\begin{abstract}
In order to ensure the just-in-time production and the highest utilization rate of jig frame resources in the construction process of the segmented hull workshop, a mathematical model of the scheduling of the segmented hull workshop based on the time and space coupling constraints was established by taking the average utilization rate of jig frame resources of the segmented workshop as the objective function. Aiming at the shortcomings of traditional genetic algorithm, a simulated annealing genetic algorithm (GASA) is designed. Finally, the actual production data of a shipyard was used to verify the feasibility and rationality of the proposed algorithm in solving the scheduling problem of segmented workshop with placenta resource as the bottleneck resource, by comparing the average resource utilization rate of placenta and the number of segments of untimely placenta with other scheduling schemes.
\end{abstract}

Index Terms - Hull segment workshop, Jig frame resources, Improved genetic algorithm, Production scheduling

\section{INTRODUCTION}

Ship building is a systematic and complicated project with long production cycle and strict time sequence of each station [1]. As a key intermediate product in ship construction, the manufacturing site of segment is mainly the jig frame in the hull segment workshop. However, the standard operating cycle of different types of segments varies greatly, the idea of hull subdivision construction is adopted in the process of segment construction, that is, to ensure that similar types of segments are manufactured in the same area as far as possible, so as to facilitate the management of field production and the stability of production rhythm.

In the actual production activities, hull segment workshop scheduling is usually rely on site management experience in scheduling, tend to tire rack to chaos, you choose position insufficient resource utilization and segmented tardiness situation [2], this paper uses the simulated annealing genetic algorithm for segmentation are applied to solve the mathematical model of the workshop scheduling problem in the meet time constraints and position resource constraints, determine the similar type of piecewise optimized scheduling scheme in the same area, achieve the expected effect of rational utilization of jig frame resource, so as to improve production efficiency.

\section{PROBLEM DESCRIPTION}

The particularity of segmental construction determines that site resources become bottleneck resources in manufacturing

Fei LI, School of Naval Architecture \& Ocean Engineering, Jiangsu University of Science and Technology, Zhenjiang, Jiangsu, China

XiaoPing MA, Professor, School of Naval Architecture \& Ocean Engineering, Jiangsu University of Science and Technology, Zhenjiang, Jiangsu, China process [1]. Therefore, the scheduling of the segmental workshop is based on the idea of hull separation construction under the restriction of the jig frame resources. Response to the advance medium-term schedule plan to meet the pull-type planning needs of each segment, maximize the utilization of the bottleneck resource of fig frame position in a certain period of time, thus controlling the rhythm of segment production to meet the needs of lean shipbuilding.

In the process of hull production, segments of similar type and similar standard operating cycle are often produced simultaneously on several tires of the same working line. In addition to selecting the appropriate jig frame for manufacturing, each segment needs to be completed within the start time and end time of the large group specified in the medium-term schedule, but the resources of the jig frame are limited[2]. On the one hand, the placement of jig frame will affect the start production time of the segments, on the other hand segment of occupy jig frame resources will in turn affect the jig site occupancy and release, jig resources and production time of these two constraints coupling each other, form the hull segment workshop scheduling problem,its essence is to make infinite use of the limited jig frame resources in the timeline[3].

\section{MODEL BUILDING}

\section{A. Hypothesis of the model}

(1) Each segment occupies only one jig frame resource.

(2) Before scheduling, all the resources of the jig frame are released.

(3) All the basic units of time in the mathematical model are days.

(4) The time of entering and exiting the jig frame of segments is recorded as the time of starting and ending the production of segments.

\section{B. Construction of objective function and constraint conditions}

Suppose there are currently $m$ segments that need to be arranged on $n$ jig frame, where the set of segments is $S=\left\{S_{1}, S_{2 x} S_{3 x \ldots} S_{m}\right\}$ and the set of jig frame is $P=\left\{P_{1}, P_{2}, P_{a}, \ldots P_{n}\right\}$.The attribute of each segment is $S_{i}=\left\{l_{i}, w_{i}, z_{i}, E S T_{i}, L S T_{i x}, L F T_{i} x T_{i}, P S T_{i}, P E T_{i}\right\}$, Among them, $l_{i}$ is the length of the segment $i, w_{i}$ is the width of the segment $i, z_{i}$ is the weight of the segment $i . E S T_{i}$ is the earliest start time of segment $i$, corresponding to the group end time in the schedule plan; $L S T_{\mathrm{i}}$ is the latest start time, corresponding to the large group start time in the schedule plan; $L F T_{i}$ is the latest end time of segment $i$, corresponding to the large group end time in the schedule plan; $L F T_{i}$ is the standard operation cycle of segment $i$. 
$P S T_{\mathrm{i}}$ is the plan start time of segment $i$ and $P E T_{\mathrm{i}}$ is the plan end time of segment $i$.

The property of each jig frame site $j$ is $P_{j}=\left\{L_{j}, W_{j}, Z_{j j}\right\}$, where $L_{j}$ is the length of site $j, W_{j}$ is the length of site $j$ and $Z_{j}$ is the lifting load weight corresponding to site $j$. Then the model of scheduling problem of hull segment workshop is as follows:

The objective function: The average resource Utilization $U$ of the site is the highest

The constraint conditions:

$$
\begin{gathered}
E S T_{\mathrm{i}} \leq P S T_{\mathrm{i}} \leq L S T_{\mathrm{i}} \\
P E T_{\mathrm{i}} \leq L F T_{\mathrm{i}} \\
S T_{\mathrm{i}}+P S T_{\mathrm{i}}=P E T_{\mathrm{i}} \\
l_{\mathrm{i}}<L_{j} \\
w_{\mathrm{i}}<W_{j} \\
z_{\mathrm{i}}<Z_{j}
\end{gathered}
$$

Formula (2) indicates that the planning start time of the segment should be between the end time of the group and the start time of the group, Formula (3) indicates that the planning end time of the segment is less than the group end time of the segment, Formula (4) indicates that the planning time of the segment is the standard operating cycle of the segment, Formula (5), Formula (6), Formula (7) indicate that the length, width and weight of the segments entering the jig frame site need to meet the requirements of the frame site length, width and crane load.

Input of the model:

(1) Basic information of all segments includes length, width, weight, end time of group, start time of large group, end time of large group and standard operating cycle.

(2)Basic information of all jig frame sites includes length, width and crane load of jig frame.

Output of the model:

The planning start time, the planning end time and the selected fig frame for each segment.

\section{ALGORITHM DESIGN}

In order to avoid premature convergence of the traditional genetic algorithm into local optimal solution and improve the efficiency of the algorithm[4], a simulated annealing operator is inserted between the chromosome crossover operator and the selection operator of the genetic algorithm. By calculating the simulated annealing replacement probability and comparing it with the random number, some possible optimal solutions are retained. The flow chart of the algorithm is shown as follows:

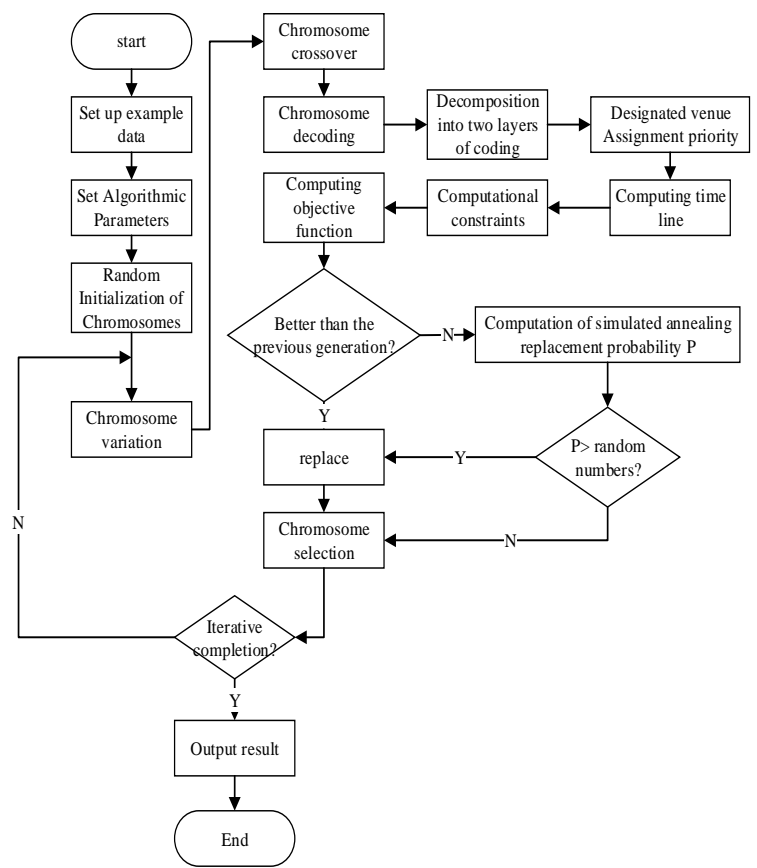

Figure 1 Flow chart of simulated annealing genetic algorithm

\section{A. Chromosome coding}

In this paper, the scheduling problem of segment workshop can be summarized as the problem of making sequence of each segment and the problem of choosing the jig frame of each segment. To solve this problem, we divide the gene strings on the same chromosome into two kinds, one is based on segmented priority sequence, the other is based on jig frame selection. Finally, a double-layer coding scheme based on two gene strings is obtained. The two gene strings have the same length and are all decimal coding schemes.

In order to better understand the double-layer coding scheme in this paper, for example, when six segments are arranged on three placentas, the sequencing gene strings can be expressed as $[3,4,1,6,5,2]$, and the placenta selection gene strings can be expressed as $[3,2,1,1,3,2]$. As shown in the figure below, the two gene strings correspond to each other one by one. The number of gene strings in the segment sequence is arranged in the priority order of all segments. The number of the jig frame sequence corresponding to each segment is the jig frame site selected by the segment.

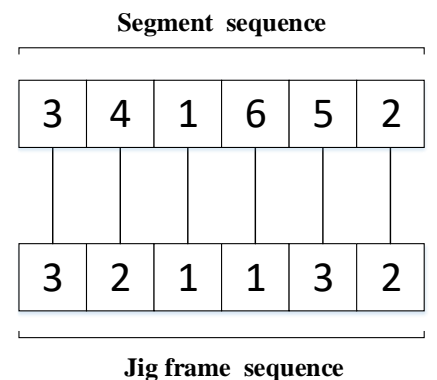

Fig 2 Simulated Annealing Genetic Algorithms Coding Scheme

\section{B. Fitness function}

In the iteration process of each generation, the search path of the genetic algorithm is determined according to the fitness value of the individual. The fitness function of this design 
takes the negative reciprocal form of the objective function, namely [5]:

$f=-\frac{1}{\max U}$

In the formula, $U$ is the objective function, that is, the average utilization rate of resources at the site. When it is the largest, the fitness function $f$ is the largest.

\section{Mutation operation}

Because of the double-layer coding scheme adopted in this design, it is necessary to mutate the two-layer coding separately. For segment priority coding, this design uses two-point mutualism method to mutate, i.e. random exchange of two genes in chromosome; for jig frame site selection coding, single-point mutation method is used to mutate, i.e. random selection of one gene in chromosome and its variation.

\section{Crossover operation}

Like the crossover operation of jig frame site selection coding, segment priority coding first randomly selects two chromosomes, then randomly generates two natural numbers A1 and A2 which are not larger than the coding length. Finally, the gene fragments A1 to A2 in the two chromosomes are crossed. In order to ensure that there is no conflict between the crossed chromosomes, the complements of the crossed fragments need to be extracted and randomly inserted into the non-crossed fragments.

\section{E. Simulated annealing operation}

In order to avoid premature convergence of the genetic algorithm and thus obtain the local optimal solution, this paper introduced the Metropolis acceptance criterion of simulated annealing algorithm, and its basic process is as follows:

(1) Determine the solution E optimized by traditional genetic algorithm at temperature;
(2) If the solution is better than the solution at the previous temperature, the new solution will be accepted, otherwise the solution will be changed (3);

(3) Replacement probability $P$ of simulated annealing when calculating temperature $T$;

$P=e^{\frac{d E}{k T}}$

(4) Comparing the replacement probability with the random number of the interval $[0,1]$, if so, the new solution will be accepted, otherwise (1).

\section{F. Selection operation}

The selection method of this design is roulette operation, that is, the probability of each individual being selected in the population is proportional to the value of fitness function. If there are $n$ individuals and the fitness value of each individual $i$ is $F_{i}$, then the probability of the individual being selected is:

$$
P_{i}=\frac{F_{i}}{\sum_{1}^{n_{1}} F_{i}}
$$

\section{VERIFICATION AND ANALYSIS}

\section{A. Examples of Shipyard Verification}

In order to verify the feasibility and rationality of the proposed genetic algorithm based on simulated annealing for solving the scheduling problem of ship hull segmented workshop, taking 50 double bottom and side segments of a large bulk carrier as an example, the standard operation cycle of the 50 segments is mostly 7 to 20 days, and the size of the segmented operation line in the workshop is $200 \mathrm{~m} * 32 \mathrm{~m}$, with a total of $20 \mathrm{jig}$ frame positions. Among them, there are 13 jig frame positions of $16 * 14$ and 7 jig frame positions of $14 * 12$. The specific information is shown in Tables 1 and 2 below.

Tab.1 Table of Basic Elements of Segmented Information

\begin{tabular}{lllllllll}
\hline No. & $\begin{array}{l}\text { Length } \\
\text { /m }\end{array}$ & Width/m & Weight/t & $\begin{array}{l}\text { Earliest } \\
\text { start time }\end{array}$ & $\begin{array}{l}\text { Latest start } \\
\text { time }\end{array}$ & $\begin{array}{l}\text { Latest end } \\
\text { time }\end{array}$ & $\begin{array}{l}\text { Standard } \\
\text { operating } \\
\text { cycle }\end{array}$ & $\begin{array}{l}\text { Carriage } \\
\text { time point }\end{array}$ \\
\hline 1 & 13.5 & 12 & 65 & $7 / 1 / 2018$ & $7 / 5 / 2018$ & $7 / 25 / 2018$ & 20 & $8 / 21 / 2018$ \\
2 & 6.4 & 3.5 & 30 & $7 / 1 / 2018$ & $7 / 3 / 2018$ & $7 / 13 / 2018$ & 7 & $8 / 9 / 2018$ \\
3 & 6.4 & 4 & 35 & $7 / 1 / 2018$ & $7 / 3 / 2018$ & $7 / 14 / 2018$ & 7 & $8 / 10 / 2018$ \\
4 & 13.5 & 12 & 70 & $7 / 1 / 2018$ & $7 / 4 / 2018$ & $7 / 30 / 2018$ & 25 & $8 / 26 / 2018$ \\
5 & 13 & 11 & 50 & $7 / 1 / 2018$ & $7 / 3 / 2018$ & $7 / 13 / 2018$ & 10 & $8 / 9 / 2018$ \\
$\ldots$ & $\ldots$ & $\ldots$ & $\ldots$ & $\ldots$ & $\ldots$ & $\ldots$ & $\ldots$ \\
46 & 13 & 10 & 55 & $8 / 5 / 2018$ & $8 / 8 / 2018$ & $8 / 26 / 2018$ & 15 & $9 / 18 / 2018$ \\
47 & 13.5 & 12 & 70 & $8 / 6 / 2018$ & $8 / 8 / 2018$ & $8 / 19 / 2018$ & 12 & $9 / 11 / 2018$ \\
48 & 13 & 9.9 & 60 & $8 / 6 / 2018$ & $8 / 8 / 2018$ & $8 / 24 / 2018$ & 15 & $9 / 16 / 2018$ \\
49 & 12.6 & 10 & 65 & $8 / 7 / 2018$ & $8 / 9 / 2018$ & $8 / 25 / 2018$ & 15 & $9 / 17 / 2018$ \\
50 & 14 & 12 & 85 & $8 / 9 / 2018$ & $8 / 11 / 2018$ & $8 / 22 / 2018$ & 10 & $9 / 14 / 2018$ \\
\hline
\end{tabular}


Tab.2 Table of Basic Elements of Jig Frame Position Information

\begin{tabular}{llll}
\hline $\begin{array}{l}\text { Jig frame } \\
\text { Position }\end{array}$ & Length/m & Width/m & $\begin{array}{l}\text { Crane load } \\
/ \mathrm{t}\end{array}$ \\
\hline 1 & 16 & 14 & 100 \\
2 & 16 & 14 & 100 \\
3 & 16 & 14 & 100 \\
$\ldots$ & $\ldots$ & $\ldots$ & $\ldots$ \\
18 & 14 & 12 & 80 \\
19 & 14 & 12 & 80 \\
20 & 14 & 12 & 80 \\
\hline
\end{tabular}

In the programming environment of MATLAB R2014b, the parameters of genetic algorithm are set: initial population number $P=200$, iteration times $M=200$, mutation probability $P_{\mathrm{m}}=0.1$ and crossover probability $P_{C}=0.8$. Simulated annealing algorithm parameters: cooling coefficient $q=0.98$, initial temperature $T_{0}=6000$, initial temperature $T_{\theta}=20$, input data in Tables 1 and 2, get the schedule of all segments, as shown below.

Tab.3 Segmented Scheduling Schedule

\begin{tabular}{|c|c|c|c|}
\hline NO. & $\begin{array}{l}\text { Planned start } \\
\text { time }\end{array}$ & $\begin{array}{l}\text { Planned } \\
\text { time }\end{array}$ & Site no. \\
\hline 1 & $2018 / 7 / 1$ & $2018 / 7 / 20$ & 11 \\
\hline 2 & $2018 / 7 / 1$ & $2018 / 7 / 7$ & 6 \\
\hline 3 & $2018 / 7 / 1$ & $2018 / 7 / 7$ & 15 \\
\hline 4 & $2018 / 7 / 1$ & $2018 / 7 / 25$ & 16 \\
\hline 5 & $2018 / 7 / 1$ & 2018/7/10 & 3 \\
\hline ... & $\cdots$ & $\ldots$ & $\ldots$ \\
\hline 46 & $2018 / 8 / 6$ & $2018 / 8 / 20$ & 6 \\
\hline 47 & $2018 / 8 / 6$ & 2018/8/17 & 4 \\
\hline 48 & $2018 / 8 / 8$ & $2018 / 8 / 22$ & 14 \\
\hline 49 & $2018 / 8 / 7$ & $2018 / 8 / 21$ & 18 \\
\hline 50 & $2018 / 8 / 9$ & $2018 / 8 / 18$ & 2 \\
\hline
\end{tabular}

In order to show clearly the situation of choosing the placement site for each segment, the Gantt chart with the number of the placement site as the ordinate coordinate and the dispatching day as the abscissa was obtained by using Matlab and the daily utilization map of site resources with placement number as abscissa and time as ordinate.

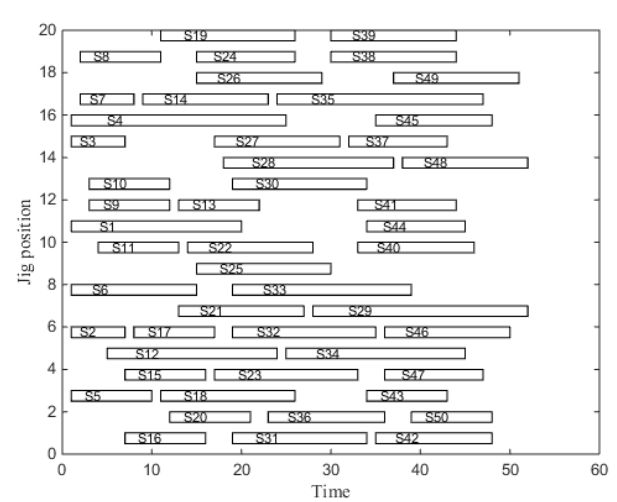

Fig 3 Scheduling Gantt Chart

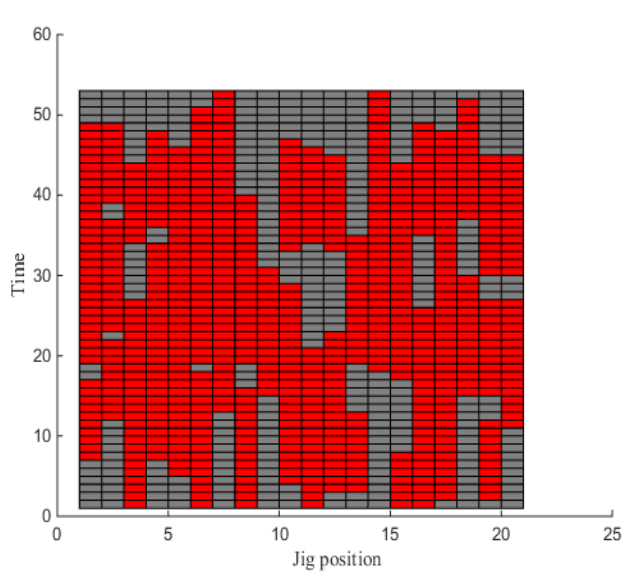

Fig 4 Resource Utilization Map of Platform

\section{B. Result comparison and analysis}

According to Gantt chart and resource utilization chart, the scheduling interval of the whole schedule is $\mathbf{5 2}$ days from July 1 to August 22. The utilization rate of fetal position resources reaches $100 \%$ on July 19 and July 20 . The utilization rate of jig frame position resources from July 21 to July 24 also exceeds $90 \%$. With the passage of scheduling time and the reduction of production segments, the utilization rate of jig frame position resources gradually decreases. In addition, by comparing with other scheduling processes, it is found that:

(1) Simulated annealing genetic algorithm (GASA) can get the optimal solution when the number of iterations reaches 110 or so, and the iterative curve is better than the traditional genetic algorithm, and the efficiency of solution is significantly improved, as shown in Fig. 5.3

(2) Simulated annealing genetic algorithm (GASA) is superior to general genetic algorithm (GA) and actual ship enterprise scheduling scheme in terms of average utilization rate, number of delayed segments and maximum utilization rate of site, as shown in Table 4.

(3) The scheduling results of all segments obtained by this scheduling scheme can basically be arranged according to the corresponding mid-calendar plan, which can ensure that the birth sequence of segments can be consistent with the order of the carrying plan. By comparing with the loading time points of each segment, it is found that the waiting time of each segment is about 3 to 6 days, which can meet the needs of balanced production, except for the painting outfitting and buffer time after the segment departure from the production site. 
The above results show that the improved genetic algorithm proposed in this paper can effectively solve the scheduling problem of ship hull segmental workshop, can produce a more reasonable segmented scheduling schedule in a relatively short time, can effectively improve the utilization rate of jig frame position resources, reduce the number of delayed segments, and achieve the desired goal. It proves that the improved algorithm is feasible.

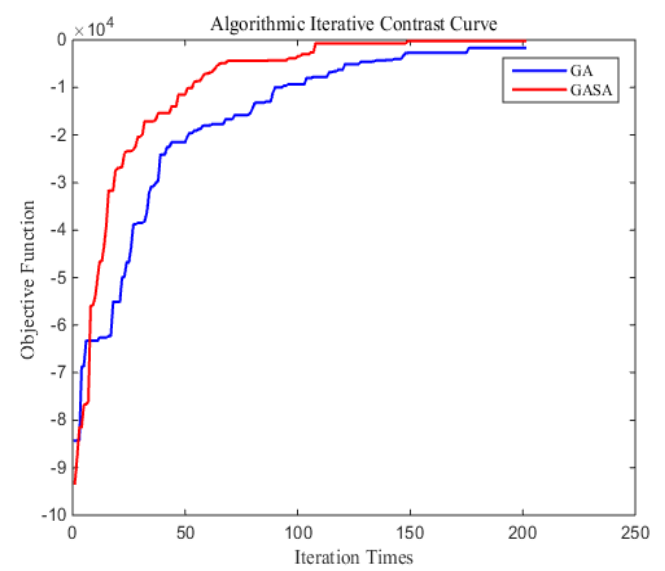

Fig 5 Comparison of GA and GASA Iterative Curves

Tab.4 Scheduling strategy comparison table

\begin{tabular}{llll}
\hline $\begin{array}{l}\text { Scheduling } \\
\text { strategy }\end{array}$ & $\begin{array}{l}\text { Average } \\
\text { Utilization } \\
\text { Rate }\end{array}$ & $\begin{array}{l}\text { Sumber } \\
\text { deferred } \\
\text { segments }\end{array}$ & $\begin{array}{l}\text { of } \\
\text { Site } \\
\text { Utilization } \\
\text { Rate }\end{array}$ \\
\hline $\begin{array}{l}\text { Enterprise } \\
\text { scheduling }\end{array}$ & $61 \%$ & 5 & $75 \%$ \\
GA & $64.2 \%$ & 6 & $85 \%$ \\
GASA & $68.6 \%$ & 2 & $100 \%$ \\
\hline
\end{tabular}

\section{CONCLUSION}

In this paper, a simulated annealing genetic algorithm is designed to solve the scheduling problem of hull segment shop based on time and space constraints. The feasibility and rationality of this method are verified by a shipyard example. The results show that the algorithm can effectively improve the utilization rate of the bracket resources in the segment shop on the basis of guaranteeing the start time and completion time of the segment, thus improving the utilization rate of the jig frame resources in the segment shop. At the same time, this paper also provides research ideas for the related segment workshop scheduling problem.

\section{REFERENCES}

[1] Hena, Dong Zhu. Application of Advanced Planning Management System for Ship Construction [J]. Science and Technology Style, 2014 (14).

[2] Ma Shaohui, Lu Chunxia. Dynamic Spatial Scheduling Algorithms for Irregular Segmentation of Ships [J]. Operations Management, 2016 (06).

[3] Research on Segmented Workshop Scheduling Method for Hull Surface Based on Spatio-temporal Constraints [J].Modern Manufacturing Engineering, 2017(9).

[4] Qi Dongxing. Design of External Ballistic Test and Study of Sensitivity Analysis Method for Electromagnetic Rail Gun [D]; Harbin University of Technology, 2017
[5] Deng Zhiyan, Chen Zhikun. Path planning of mobile robots based on improved genetic algorithm [J]. Mechanical design and manufacturing, 2010 (7): 147-14

Fei LI, School of Naval Architecture \& Ocean Engineering , Jiangsu University of Science and Technology, Zhenjiang, Jiangsu, China.

XiaoPing MA, Professor, School of Naval Architecture \& Ocean Engineering, Jiangsu University of Science and Technology, Zhenjiang, Jiangsu, China 